\title{
Pulsating stars in the open cluster NGC 1817
}

\author{
T. Arentoft ${ }^{1,2}$, L. M. Freyhammer ${ }^{2,3}$, M.Y. Bouzid ${ }^{2}$, C. Sterken ${ }^{2,4}$, \\ S. Frandsen ${ }^{2}$ \\ ${ }^{1}$ Aarhus Universitet, Ny Munkegade, DK-8000 Aarhus C, Denmark \\ ${ }^{2}$ Vrije Universiteit Brussel, Pleinlaan 2, B-1050 Brussels, Belgium \\ ${ }^{3}$ Koninklijke Sterrenwacht, Ringlaan 3, B-1180 Brussels, Belgium
}

\begin{abstract}
We report on new CCD observations ${ }^{5}$ of the open cluster NGC 1817, obtained as part of a program to search for pulsating stars in clusters. In a previous study, several $\delta$ Sct stars were found in NGC 1817 and our observations confirm that NGC 1817 is a special cluster, as we detected 19 variable stars. Twelve of these are most likely $\delta$ Sct stars of which 11 seem to be cluster members. One is also an eclipsing binary.
\end{abstract}

\section{Introduction}

The $\delta$ Sct stars are one of the most promising classes of stars for asteroseismology (the use of pulsational frequencies to constrain the stellar models) in stars near or on the main-sequence. They oscillate in mainly radial and non-radial pmodes with periods of the order of hours. Most are multiperiodic with mmag amplitudes. The potential for asteroseismology of these A-F stars lies in the multiperiodicity, since each frequency provides physical information about the star and can be used in tests of the stellar models. These become more stringent if parameters such as distance and age can be obtained independently, as can be done in a cluster. STACC (Frandsen 1992) is an observing network with the purpose of using small telescopes for observing pulsating stars in open clusters. Because most small telescopes are situated in the northern hemisphere, the focus in recent years has been on finding northern clusters with many shortperiod variables within a small field-of-view (FoV), making CCD observations very efficient. This search has now borne fruit since two outstanding clusters have been found. One is NGC 1817, the other is NGC 7062 where $13 \delta$ Sct stars were detected (Freyhammer et al. 2001). This paper is a progress report on the study of NGC 1817 .

\footnotetext{
${ }^{4}$ Research Director, Belgian Fund for Scientific Research (FWO)

${ }^{5}$ Based on observations obtained at the Nordic Optical Telescope, operated on the island of La Palma, jointly by Denmark, Finland, Iceland, Norway and Sweden, in the Spanish Observatorio del Roque de los Muchachos of the Instituto de Astrofisíca de Canarias.
} 


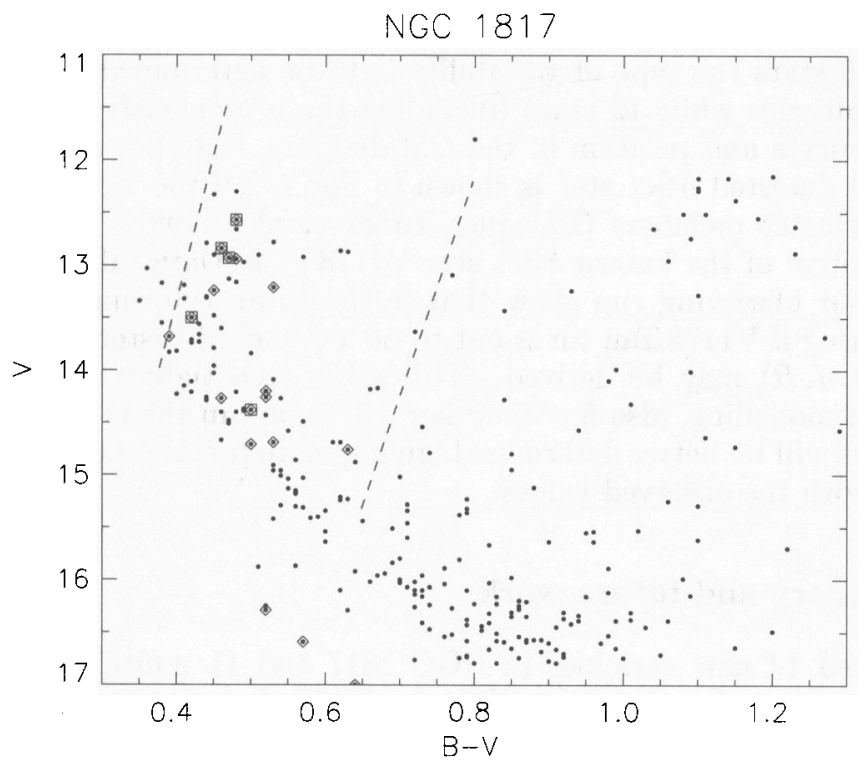

Figure 1. Colour-magnitude diagram for NGC 1817 (Mermilliod 1995). The $\delta$ Sct instability strip is indicated by the dashed lines (Breger 2000). The detected variables are marked with $\diamond$-symbols, five previously-known variables are also marked with a square.

\section{The target cluster and the new observations}

NGC 1817 was originally chosen as a STACC target because its MS turn-off is located in the $\delta$ Sct instability strip. As shown in Fig. 1, the cluster has a large number of potential $\delta$ Sct stars; because they are among the brightest members, good photometry can easily be obtained. Also, NGC 1817 is non-crowded, so we could now observe about 500 stars without degradation of the photometry due to crowding effects. Based on two nights of CCD observations, Frandsen \& Arentoft (1998) found 7 potential $\delta$ Sct stars in the cluster.

The new data were obtained by one of us (M. Y. Bouzid) with the Nordic Optical Telescope in 2002 December. The aim was to obtain time series to look for pulsating stars and to obtain uvby data for deriving physical parameters. We had excellent weather during all 5 nights awarded to the project. The FoV is about $6^{\prime} \times 6^{\prime}$ so to include more stars in the search, two neighbouring fields were observed with an overlap of $2^{\prime}$. At present we can show results from the time series only, since the Strömgren data are still being analysed.

\section{Light curves of pulsating stars}

The goals of the observations were to obtain more data on the known variable stars and to search for new variables in a larger field. These goals have been fully met as we find 19 variables, of which only 5 were previously known. For 
5 of the 19 stars the type of variability is to be determined, 2 are most likely eclipsing binaries while 12 stars (including the 5 previously known), based on the light curves and position in the CM-diagram, are $\delta$ Sct stars. An example of a newly detected $\delta$ Sct star is shown in Fig. 2. Of the $12 \delta$ Sct stars, 11 are probable cluster members (Balaguer-Núnez et al. 1998). In Fig. 3 we show the light curve of the known $\delta$ Sct star V1178 Tau. Data taken during the last hours of our observing run show that it is also an eclipsing binary. It could be interesting if V1178 Tau turns out to be a detached system because absolute elements $(M, R)$ may be derived. This gives even tighter constraints on the theoretical modelling, also for the other $\delta$ Sct stars in the cluster as their radii and masses will be better determined, giving more precise theoretical periods to compare with the observed values.

\section{Summary and future work}

We detected 14 new variables in NGC 1817 and 11 multiperiodic $\delta$ Sct stars belonging to the cluster are now known. This gives good prospects for asteroseismic studies because common parameters such as age and distance can be imposed on the models of all the pulsating stars. Based on these results we plan to launch a multi-site campaign in order to obtain sufficient time-series data to allow detailed tests of stellar evolution models for $2 \mathrm{M}_{\odot}$ stars. However, before doing so we wish to finish the analysis of the uvby data already obtained and to perform spectroscopic observations of the interesting stars in order to check for binarity (and in the case of V1178 Tau, investigate the binarity) as well as to determine $v \sin i$ and abundances.

Acknowledgments. TA acknowledges financial support from The Danish Natural Science Research Council, partly through the project "Stellar structure and evolution - new challenges from ground and space observations" carried out at Aarhus University and Copenhagen University. LMF and CS acknowledge support from the project IUAP P5/36 financed by the Belgian State, Federal Office for Scientific, Technical and Cultural Affairs. MYB and CS acknowledge financial support from the Belgian Fund for Scientific Research (FWO).

\section{References}

Balaguer-Núnez, L., Tian, K.P., Zhao, J.L. 1998, A\&AS, 133, 387

Breger, M. 2000, ASP Conf. Ser., Vol. 210, Delta Scuti and Related Stars, eds. M. Breger \& M. Montgomery, (San Francisco: ASP), 3

Frandsen, S. 1992, Delta Scuti Star Newsletter (Univ. of Vienna), 5, 12

Frandsen, S., Arentoft, T. 1998, A\&A 333, 524

Freyhammer, L.M., Arentoft, T., Sterken, C. 2001, A\&A 368, 580

Mermilliod, J.-C. 1995, in Information and On-Line Data in Astronomy, ed. D. Egret \& M. A. Albrecht, (Dordrecht: Kluwer), 127 


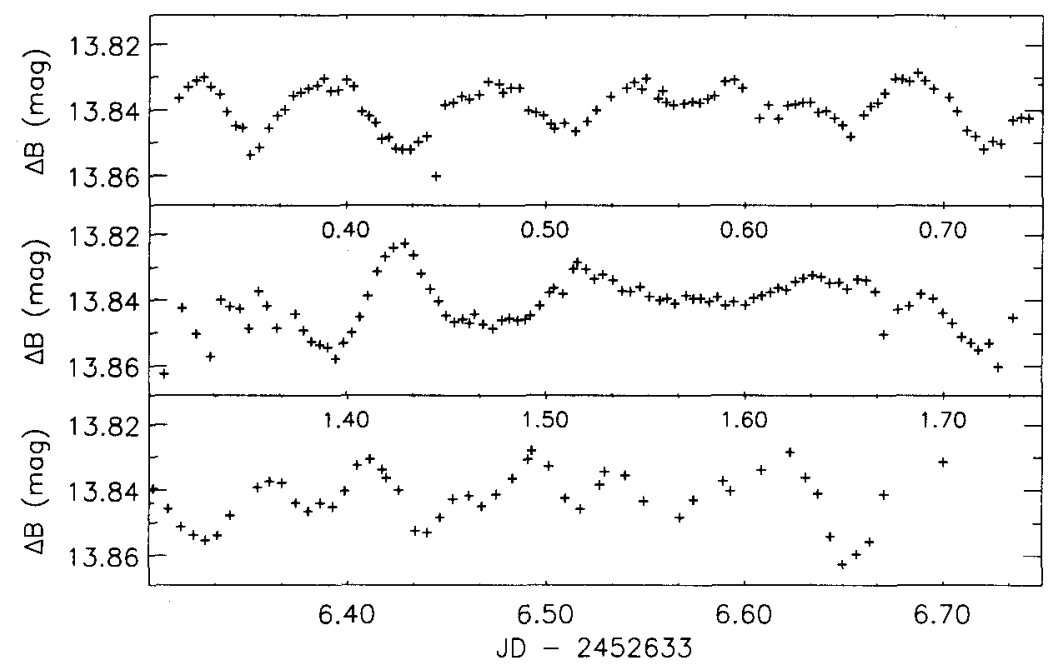

Figure 2. A new multiperiodic $\delta$ Sct star detected from our data. The data are less abundant during the last night because alternating $B$ and $V$ images were obtained.

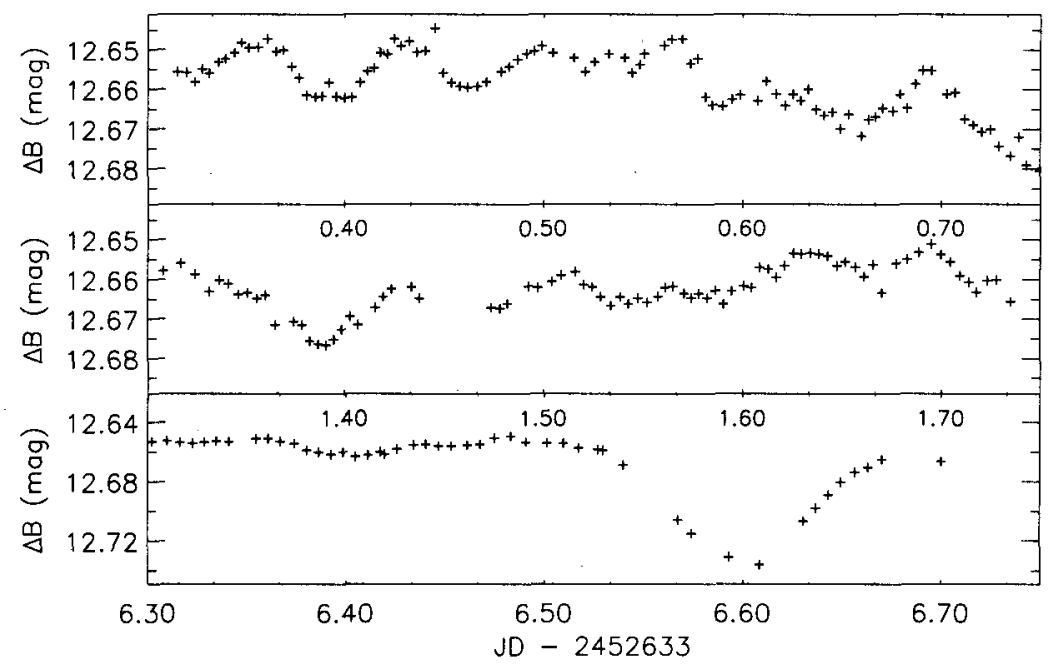

Figure 3. V1178 Tau, a previously known $\delta$ Sct star which is in fact also an eclipsing binary (note that the bottom panel has a different $y$-scale than the two top panels). 


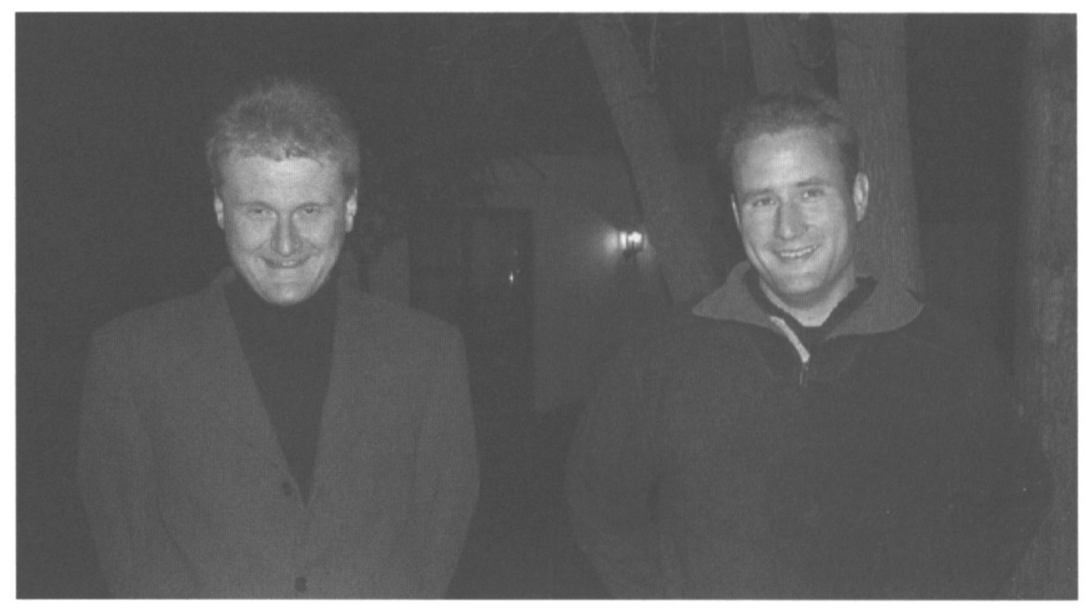

Gerald Handler and Torben Arentoft

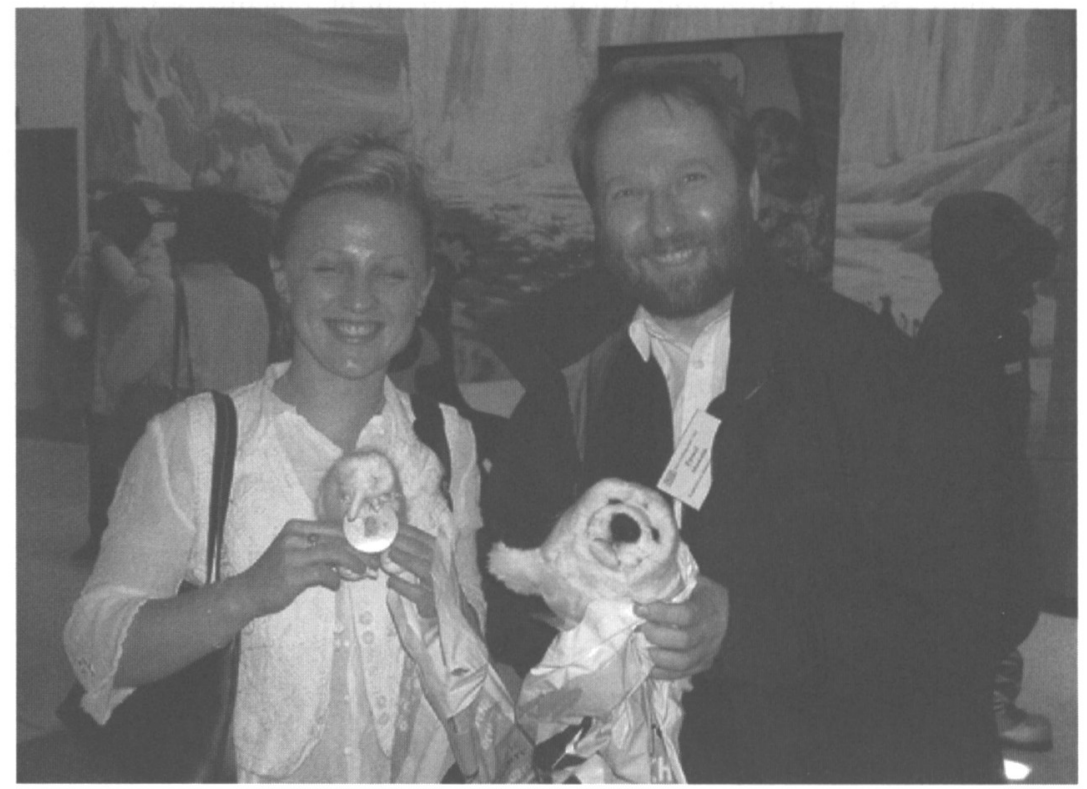

Jagoda Daszyńska-Daszkiewicz and Pawel Moskalik 